GLYCAEMIC VARIABILITY IN PREGNANT WOMEN WITH

\title{
GESTATIONAL DIABETES
}

\section{Nida OZTOP' , Selda Celik GEDIK², Elif BAGDEMIR², Yildiz TUTUNCU², Cemile IDIZ², Ayse KUBAT UZUM ${ }^{2}$, ilhan SATMAN ${ }^{2}$, Nevin DINCCAG ${ }^{2}$}

'Istanbul University, Istanbul Faculty of Medicine, Internal Medicine Division, Istanbul-Turkey

${ }^{2}$ Istanbul University, Istanbul Faculty of Medicine, Endocrinology and Metabolism Division, Istanbul-Turkey

\section{INTRODUCTION}

The accurate and comprehensive assessment of glycemic control in pregnants with gestational diabetes(GDM) is important for preventing fetal complications. We aimed to determine glycaemic variation on women with GDM with using a continuous glucose monitoring system (CGMS) and to investigate the relationship between reflecting glucose markers such as Glycated hemoglobin (A1C), Fructosamine (FRM) and 1,5-Anhydroglucitol (1,5-AG).

\section{MATERIAL AND METHOD}

31 women with GDM on diet therapy only (mean age $31,9 \pm 6,9$ yrs, gestational week $\geq 35$ ) were recruited from outpatient clinic. Those patients were screened by self monitoring blood glucose(SMBG) and monitored for three consecutive days to obtain mean daily glucose data; glycaemic fluctuations were evaluated using postprandial incremental Area Under Curve(AUC) and percent of Mean Absolute Difference(MAD\%). Venous blood samples were collected to measure A1C, FRM and 1,5-AG.

Table-1: Demographic and clinical characteristic features of the study group

\begin{tabular}{|c|c|c|c|c|c|c|}
\hline & & Median & Min-Max & Ave. \pm s.s & $\mathbf{n}$ & $\mathrm{n} \%$ \\
\hline \multicolumn{2}{|l|}{ Number of patient ( $n$ ) } & & & & 31 & \\
\hline \multicolumn{2}{|l|}{ Age (year) } & 32 & $20-47$ & $31,9 \pm 6,9$ & & \\
\hline \multirow[t]{2}{*}{ Family history of diabetes } & yes & & & & 20 & $64,5 \%$ \\
\hline & no & & & & 11 & $35,5 \%$ \\
\hline \multicolumn{2}{|l|}{ Gestational Age } & 36 & $35-38$ & $35,8 \pm 0,7$ & & \\
\hline \multicolumn{2}{|l|}{ Body Mass Index (kg/m²) } & 26,4 & $17,3-44,1$ & $26,2 \pm 5,9$ & & \\
\hline \multicolumn{2}{|c|}{ Weight before pregnancy $(\mathrm{kg})$} & 66 & $46-110$ & $\begin{array}{c}67,0 \pm 15 \\
0\end{array}$ & & \\
\hline \multicolumn{2}{|l|}{ Weight during pregnancy $(\mathbf{k g})$} & 13 & $5-19$ & $12,2 \pm 3,5$ & & \\
\hline \multirow{4}{*}{ Obstetric examination } & Polyhydroamnios & & & & 2 & $6,4 \%$ \\
\hline & Oligohydroamnios & & & & 1 & $3,2 \%$ \\
\hline & $\begin{array}{l}\text { Doubt of trisomy } \\
21\end{array}$ & & & & 2 & $6,4 \%$ \\
\hline & Cleft palate-lip & & & & 1 & $3,2 \%$ \\
\hline Obstetric history & yes & & & & 16 & $51,6 \%$ \\
\hline \multirow[t]{2}{*}{$\begin{array}{l}\text { (History of abortus, } \\
\text { stillbirth or macrozomia) }\end{array}$} & no & & & & 15 & $48,4 \%$ \\
\hline & yes & & & & 4 & $12,9 \%$ \\
\hline Additional Dissease & no & & & & 27 & $89,1 \%$ \\
\hline
\end{tabular}

Table-2: Biochemical parameters

$n: 31$

\begin{tabular}{|c|c|c|c|}
\hline & Median & Min-Max & Ave.土s.s. \\
\hline $\mathrm{HbA}_{1 \mathrm{c}}(\%)$ & 5,0 & $4,4-5,8$ & $5,0 \pm 0,3$ \\
\hline Fructozamine ( $\mu \mathrm{mol} / \mathrm{L})$ & 2,2 & $1,5-2,6$ & $2,1 \pm 0,2$ \\
\hline 1,5-Anhydroglucitol ( $\mathrm{ng} / \mathrm{mL})$ & 15,4 & $3,8-27,4$ & $17,0 \pm 4,9$ \\
\hline Triglyceride(mg/dL) & 186 & $86-401$ & $204,3 \pm 72,3$ \\
\hline HDL (mg/dL) & 64 & $40-110$ & $65,5 \pm 17,3$ \\
\hline LDL (mg/dL) & 164 & $79-230$ & $151,5 \pm 38,7$ \\
\hline
\end{tabular}

Table-3: CGMS and SMBG parameters

\begin{tabular}{|c|c|c|c|}
\hline$n: 31$ & Median & Min-Max & 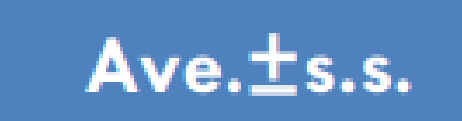 \\
\hline Highest glucose in 3 days $(\mathrm{mg} / \mathrm{dL}$ ) & 127 & $99-185$ & $131,1 \pm 22,5$ \\
\hline Lowest glucose in 3 days $(\mathrm{mg} / \mathrm{dL})$ & 52 & $40-88$ & $54,7 \pm 11,6$ \\
\hline Average glucose in 3 days ( $\mathrm{mg} / \mathrm{dL}$ ) & 85 & $72-117$ & $86,1 \pm 10,3$ \\
\hline MAD \% & 5,8 & $2,0-15,8$ & $6,7 \pm 3,1$ \\
\hline $\begin{array}{l}\text { Total excursions of gluocose in } 3 \\
\text { days }\end{array}$ & 6 & $1-16$ & $5,8 \pm 3,6$ \\
\hline AUC Above-140 mg/dL & 0,0 & $0,0-1,5$ & $0,2 \pm 0,4$ \\
\hline AUC Below-70 mg/dL & 1,0 & $0,0-3,7$ & $1,3 \pm 1,2$ \\
\hline $\begin{array}{l}\text { Mean glucose measured by } \\
\text { SMBG(mg/dL) }\end{array}$ & 81,5 & $54,8-110,1$ & $82,9 \pm 10,2$ \\
\hline
\end{tabular}

Table-4: Infant's demographic and clinical characteristic features

\begin{tabular}{|c|c|c|c|c|c|c|}
\hline $\mathrm{n}: 31$ & & Median & Min-Max & Ave.Es.s & $\mathbf{n}$ & $\mathbf{n} \%$ \\
\hline \multicolumn{2}{|c|}{ Infant Birth Weight(gr) } & 3190 & $2390-3800$ & $3142,9 \pm 366,2$ & & \\
\hline \multicolumn{2}{|c|}{ Infant Birth Length (cm) } & 48 & $42-53$ & $47,7 \pm 2,6$ & & \\
\hline \multicolumn{2}{|c|}{ Infant Head Circumference(cm) } & 34 & $32-38$ & $34,3 \pm 1,3$ & & \\
\hline \multirow{3}{*}{ Jaundice } & $\begin{array}{l}\text { Prolonged } \\
\text { jaundice }\end{array}$ & & & & 1 & $3,2 \%$ \\
\hline & $\begin{array}{l}\text { Neonatal } \\
\text { jaundice }\end{array}$ & & & & 13 & $41,9 \%$ \\
\hline & No & & & & 17 & $54,8 \%$ \\
\hline \multirow{2}{*}{$\begin{array}{l}\text { Respiratory } \\
\text { Distress }\end{array}$} & Yes & & & & 2 & $6,5 \%$ \\
\hline & No & & & & 29 & $93,5 \%$ \\
\hline \multirow{3}{*}{$\begin{array}{l}\text { Other neonatal } \\
\text { complications }\end{array}$} & $\begin{array}{l}\text { Gallbladder } \\
\text { atresia }\end{array}$ & & & & 1 & $3,2 \%$ \\
\hline & Eye infection & & & & 1 & $3,2 \%$ \\
\hline & $\begin{array}{l}\text { Neonatal } \\
\text { hypoglycaemia }\end{array}$ & & & & 0 & $0 \%$ \\
\hline
\end{tabular}

\section{RESULTS}

Prepregnancy BMI of participants was $26,2 \pm 5,9 \mathrm{~kg} / \mathrm{m}^{2}$, weight gain during pregnancy was obtained as $12,2 \pm 3,5$ kg;levels of reflecting glucose markers were measured as

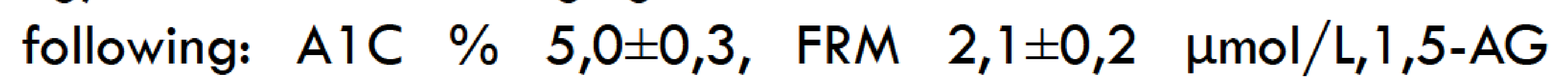
$17,0 \pm 4,9 \mathrm{ng} / \mathrm{ml}$; according the results of CGM, MAD \% was found as $\% 6,7 \pm 3.1$, the total number of fluctuations in glucose levels were counted as $5,8 \pm 3,6$. Glucose figures, measured by SMBG or CGMS were found similar $(82,9 \pm 10,2$ and $86,1 \pm 10,3 \mathrm{mg} / \mathrm{dL}$ ); I statistically, there wasn't any correlation between determinants of CGM and other glucose reflection parameters. It was realized that birth of weight and size of head circumference of babies were affected by maternal glucose levels.

\section{CONCLUSION}

Although it seems that normoglycaemia is sustained, there should be glucose variability in diabetic patients especially during pregnancy and all known glucose reflecting parameters do not show fluctuations. CGMS is an alternative method for detecting glucose variations in spite of having difficulties to afford and apply the tool. We concluded that higher glucose fluctuations were observed on pregnants with GDM who have high triglyceride at fasting and high glucose levels at 3 rd hour of OGTT at the beginning 